\title{
Senescence of mesenchymal stem cells (Review)
}

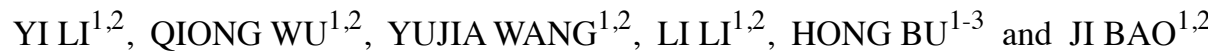 \\ ${ }^{1}$ Laboratory of Pathology, West China Hospital, Sichuan University; ${ }^{2}$ Key Laboratory of Transplant Engineering and Immunology, \\ Chinese Ministry of Health, West China Hospital, Sichuan University; ${ }^{3}$ Department of Pathology, \\ West China Hospital, Sichuan University, Chengdu, Sichuan 610041, P.R. China
}

Received February 2, 2016; Accepted January 13, 2017

DOI: $10.3892 / \mathrm{ijmm} .2017 .2912$

\begin{abstract}
Mesenchymal stem cells (MSCs) have been used in cell-based therapy for various diseases, due to their immunomodulatory and inflammatory effects. However, the function of MSCs is known to decline with age, a process that is called senescence. To date, the process of MSC senescence remains unknown as in-depth understanding of the mechanisms involved in cellular senescence is lacking. First, senescent MSCs are so heterogeneous that not all of them express the same phenotypic markers. In addition, the genes and signaling pathways which regulate this process in MSCs are still unknown. Thus, an understanding of the molecular processes controlling MSC senescence is crucial to determining the drivers and effectors of age-associated MSC dysfunction. Moreover, the proper use of MSCs for clinical application requires a general understanding of the MSC aging process. Furthermore, such knowledge is essential for the development of therapeutic interventions that can slow or reverse age-related degenerative changes to enhance repair processes and maintain healthy function in aging tissues. To further clarify the properties of senescent cells, as well as to present significant findings from studies on the mechanisms of cellular aging, we summarize these biological features in the senescence of MSCs in this scenario. This review summarizes recent advances in our understanding of the markers and differentiation potential indicating MSC senescence, as well as factors affecting MSC senescence with particular emphasis on the roles of oxidative stress, intrinsic changes in telomere shortening, histone deacetylase and DNA methyltransferase, genes and signaling pathways and immunological properties.
\end{abstract}

\section{Contents}

1. Introduction

2. Markers of senescent MSCs

Correspondence to: Professor Ji Bao, Laboratory of Pathology, West China Hospital, Sichuan University, 37 Guoxue Road, Chengdu, Sichuan 610041, P.R. China

E-mail: baoji@scu.edu.cn

Key words: mesenchymal stem cells, senescence, reactive oxygen species, telomere, gene
3. Changes in the differentiation potential of senescent MSCs

4. ROS accumulation: base of senescence

5. Intrinsic changes in senescent MSCs

6. Strategies to prevent senescence

7. Conclusion

\section{Introduction}

Although the differentiation potential of mesenchymal stem cells (MSCs) is relatively restricted compared to pluripotent stem cells such as embryonic stem cells (ESCs) and induced pluripotent stem cells (iPSCs), MSCs are more feasible and a much safer source for cell therapy in regards to the risk of transplanted stem cells forming tumors and becoming cancerous. To date, cultures of MSCs have been successfully established from bone marrow, umbilical cord blood, trabecular bone, periosteum, synovium, placenta, pancreas, adipose tissue, skin, lung and thymus $(1,2)$. MSCs have the property of self-renewal and commit to multiple cell lineages, such as bone, cartilage, adipose, muscle, tendon, stroma and neuronal cells (3), which make them overwhelmingly useful tools in tissue engineering and regenerative medicine. MSCs show immunomodulatory effects, which attenuate the functions of dendritic cells (DCs) through the suppression of the MHC II molecule and co-stimulatory molecules (4,5). MSCs were also found to be immune suppressive in mixed lymphocyte assays by inhibiting T-cell proliferation (6). They also own the ability to support parenchymal cells as a niche with which to maintain cell pools and constitutively secrete a distinct set of cytokines and growth factors for maintaining homeostasis of the microenvironment throughout their lifetime (7). Thus, MSCs have been broadly applied in the treatment of various diseases, including graft-versus-host disease (GVHD), Crohn's disease (CD), diabetes mellitus (DM), multiple sclerosis (MS), myocardial infarction (MI), liver failure, and rejection after liver transplant (8). Unfortunately, the function of MSCs is known to decline with age, a process that may be implicated in the loss of maintenance of tissue homeostasis leading to organ failure and diseases of aging (9). Therefore, the proper use of MSCs for clinical applications requires a general understanding of the MSC aging process. Understanding the molecular processes controlling MSC proliferation, senescence and commitment to specific differentiated cell lineages is not only crucial to determining the drivers and effectors of age-associated MSC dysfunction but essential for the development of therapeutic 
interventions that can slow, even reverse, age-related degenerative changes to enhance repair processes and maintain healthy function in aging tissues.

\section{Markers of senescent MSCs}

Earlier passage MSCs have been demonstrated to have better colony efficiency compared to later passage MSCs (10). The classic features characterizing the senescence phenotype of MSCs include growth arrest in the G1 phase of the cell cycle, enlarged or flattened morphology, increased expression of senescence-associated $\beta$-galactosidase (SA- $\beta$-gal) and senescence-associated lysosomal $\alpha$-L-fucosidase (SA- $\alpha$-Fuc), and surface marker alteration (11).

Senescent MSCs usually become flat and hypertrophic with constrained nuclei and granular cytoplasm resulting from accumulation of cell debris. They have an excess of actin fibers and decreased adherence to plastic surfaces. The level of autofluorescence is increased due to lipofuscin aggregation.

The colony-forming unit-fibroblast (CFU-F) assay can be used to estimate the proliferative and clonogenic potential of MSCs in culture. MSCs are plated on plastic culture dishes at a low density and cultured under conditions that allow individual CFU-F to adhere and proliferate. The number of colonies present indicates the ability to proliferate, and the senescent MSCs show a decreased level of CFU-F.

Senescence marker, SA- $\beta$-gal, is widely used in the assay of senescent cells. Histochemical detection of SA- $\beta$-gal expression by senescent cells was first published in 1995, and continues to be one of the most common assays used to assess the senescence of MSCs. The percentage of SA- $\beta$-gal-positive MSCs is obviously increased during the process of aging, which is caused by increased lysosomal activities and altered cytosolic $\mathrm{pH}(12,13)$.

Recently, SA- $\alpha$-Fuc has been identified as a novel marker of senescence that is upregulated in response to replicative, DNA damage-, and oncogene-induced senescence. Furthermore, as a marker of senescence, SA- $\alpha$-Fuc is a more sensitive and robust biomarker for cell senescence when compared with SA- $\beta$-gal at the transcriptional and enzymatic levels (14).

The International Society for Cellular Therapy (ISCT) has proposed the minimal criteria for the definition of MSCs in 2006: i) adherence to plastic; ii) expression of surface markers CD90, CD73 and CD105, in the absence of CD45, CD34, CD14, CD11b, CD79 $\alpha$, CD19 and HLA-DR; and iii) multipotent differentiation potential of chondrocytes, osteoblasts, and adipocytes under different standard conditions in vitro (15). These markers are expressed at similar levels in early-passage, young and late-passage senescent MSCs indicating that their value may be limited only to basic MSC characterization (16). Other potential positive and negative antigenic markers have been suggested to identify senescence since Stro-1 (17), CD106 (VCAM-1) (18) and CD146 (MCAM) (19) expression levels are downregulated during prolonged culture. Moreover, CD106 expression is also strongly downregulated in MSCs after differentiation in adipogenesis, osteogenesis, and chondrogenesis, suggesting that CD106 may be a marker of undifferentiated cells within expanded MSC cultures. Meanwhile, CD295 (leptin receptor) was found to increase as a function of intrinsic cellular aging, suggesting its ability to mark apoptotic cells (20). Based on the above-mentioned findings, these markers of MSCs can be classified into two categories. One category includes molecules that are stably expressed, such as CD73, CD90 and CD105 showing little association with culture history and aging status. The second group of markers, such as Stro-1 or CD106, contains molecules which show dependency on donor, culture passage or other variables including cell seeding density, cell homing or attachment properties.

\section{Changes in the differentiation potential of senescent MSCs}

MSCs are known as having high osteogenesis and adipogenesis potential, and the switch between osteogenetic and adipogenetic commitment and differentiation is mediated through numerous transcription factors and signaling pathways. The mutual exclusivity of cell fate is thought to be determined by factors such as the CCAAT/enhancer binding protein (C/EBP), as well as peroxisome proliferator-activated receptor $\gamma(\operatorname{PPAR} \gamma)$, which promote adipocyte maturation, or runt-related transcription factor/core binding factor $\alpha 1$ (RUNX2/CBFA1), an osteoblastic transcriptional mediator (21).

Some studies indicate that the osteogenic activity of MSCs deteriorates as a function of increasing lifespan, which is in line with the loss of bone-forming efficiency. This osteogenesis is attributed to the expression of RUNX2/CBFA1 through the PI3K-AKT pathway. It is an essential transcription factor for the osteogenic/chondrogenic lineages as an activator and marker of osteogenesis of MSCs $(22,23)$ and a slight decline is observed in its expression with age (24). Receptor activator of nuclear factor- $\kappa \mathrm{B}$ ligand (RANKL), which is essential for the differentiation and maintenance of osteoclasts, was observed to be highly expressed in late-passage MSCs. The transforming growth factor- $\beta$ (TGF- $\beta$ )/SMAD3 signaling pathway is critical for osteogenic differentiation and can induce ERK phosphorylation. In addition, an inhibitor of ERK was found to suppress osteogenic differentiation induced by TGF- $\beta$ alone $(25,26)$.

According to reports, the adipogenic differentiation potential of MSCs remained unchanged, became worse or was enhanced. However, there is a general agreement that the adipogenesis potential of MSCs tends to decline with consecutive passages under standard culture conditions. PPAR $\gamma$, a member of the ligand-activated nuclear receptor superfamily, is regarded as an adipogenic-specific transcription factor, inducing the transcriptional activation of many different target genes involved in lipid metabolism and adipocyte differentiation. It has been reported that expression of PPAR $\gamma$ decreases during aging, and impaired PPAR $\gamma$ and C/EBP shift the fate of MSCs toward the osteoblast lineage. The WNT/ $\beta$-catenin signaling pathway can suppress C/EBP and PPAR $\gamma$, favoring MSC differentiation to osteoblasts, and therefore are regarded as master moderators of adipogenesis and osteogenesis (27). The phosphorylation of AKT by insulin was found to suppress FOXO3 expression and activate PPAR, which reverses differentiation balance and promotes adipogenesis potential. Activation of the PPAR $\gamma$ transcription factors by agonists such as thiazolidinedione compounds may be a strategy worthy of trial for the functional expansion of MSCs. Leptin, an important paracrine signaling molecule, may modulate the reciprocal differentiation of stromal cells between the 
osteoblastic and adipogenic pathway, and is associated with aging (28). It increases proliferation, differentiation toward osteoblasts, and inhibits differentiation to adipocytes (21). However, some studies demonstrated that blockade of leptin in MSC cultures abolished induced senescence through the PI3K/AKT signaling pathway. Recent findings on glucagonlike peptide-1 (GLP-1) also indicate that it has effects on the differentiation of MSCs into osteoblasts and adipocytes. GLP-1 upregulates the activity and mRNA expression of osteoblast-specific marker, alkaline phosphatase (ALP) and the mineralization of calcium, while suppressing the expression of PPAR $\gamma$, LPL and adipocyte protein 2 (AP2) (29). Factors such as lipoprotein lipase (LPL), which are involved in the metabolism of fat, also induce the differentiation of MSCs away from an osteoblast phenotype to adipocytes (30).

\section{ROS accumulation: base of senescence}

The free radical theory of aging was first proposed in 1956 (31). Free radicals are unstable molecules that have an unpaired electron, including nitric oxide (NO), several of the reactive oxygen species (ROS), and their reactive products. ROS include superoxide anions $\left(\mathrm{O}_{2}^{-}\right)$, hydrogen peroxide $\left(\mathrm{H}_{2} \mathrm{O}_{2}\right)$, and hydroxyl radicals $(\mathrm{OH} \cdot), \sim 90 \%$ of which are generated in cells by the mitochondrial respiratory chain $(32,33)$. The NADPH Nox family of oxidases is another major source of ROS (34). Other extrinsic factors (radiation, ultraviolet light and growth factors) also cause ROS production.

Oxidative stress is defined as an imbalance between the production of free radicals/ROS and antioxidants (35). Superoxide dismutase (SOD), catalase, peroxiredoxin, thioredoxin and glutathione systems are known as antioxidative enzymes (36). Among these antioxidants, SOD is a class of enzymes that catalyzes the dismutation of superoxide into oxygen and hydrogen peroxide, which plays the most important role. Studies have found reduced SOD activity along with increasing NO, ROS, oxidized and glycated protein levels in late-passage MSCs $(37,38)$.

The markers of stress resistance, heat shock proteins (HSPs), play a central part in stem cell differentiation and self-renewal. The responsiveness of MSCs to HSPs decreases with age. Thus impaired HSPs and stress response lead to reduced protective mechanisms, proliferation and differentiation capacity (39). Ataxia telangiectasia mutated (ATM) serine threonine protein kinase is a critical enzyme in the regulation of the stress response to DNA damage, specifically double-strand DNA breaks. Loss-of-function mutations in ATM are associated with MSC senescence (40).

Nuclear factor erythroid 2-related factor 2 (NRF2) is a master regulator and transcription factor in response to oxidative stress, and activates various antioxidant responsive element (ARE)-dependent genes encoding the above-mentioned antioxidants. NRF2 has been shown to critically control MSC survival under oxidative damage. The activity of NRF2 declines during senescence $(32,37)$ while its activation induces lifespan extension of MSCs (41).

The PI3K/AKT/mTOR/FOXO3 signaling pathway is a critical regulator of stress responses. It is reported that in the presence of high levels of ROS, FOXO3 expression is activated, and inhibition of FOXO3 results in fewer apoptotic cells and better viability (42). Moreover, the p38/MAPK signaling pathways are also responsible for establishing an irreversible ROS-induced MSC senescence, and suppression of the p38/ MAPK pathway results in partial prevention.

The ongoing oxidative process leads to DNA damage, protein damage and mitochondrial dysfunction, which are interconnected and cause reciprocal transformation (Fig. 1). Defects in proteostasis commonly lead to aberrant folding, toxic aggregation and accumulation of damaged proteins, which can in turn cause cellular damage and tissue dysfunction (43). In brief, ROS accumulation in MSCs contributes to the loss of homeostasis, leading to senescence (44).

\section{Intrinsic changes in senescent MSCs}

Telomere and telomerase. Hayflick and Moorhead uncovered the fact that primary fibroblasts exhibit only a finite proliferative capacity in culture, before they exit the cell cycle in a state known as replicative senescence and the term has been coined the Hayflick phenomenon. It has been demonstrated that progressive telomeric erosion associated with the accumulation of DNA damage causes this phenomenon (45). The in vitro lifespan of MSCs reportedly ranges from 20-40 doublings (46). Telomere shortening is observed over a lifetime, and it is estimated that every year telomeres shorten by $\sim 17$ bp during aging (47). In MSCs, the average telomere length in early-passage MSCs depends on the age of the tissue donor and it has been postulated to range from 10 to $11 \mathrm{~kb}$ in cells from fetal tissues to $7 \mathrm{~kb}$ in cells from postnatal sources (48). Other studies have reported longer telomere lengths varying from 11-13 to 9-10 kb in early MSC cultures (49,50). Telomere-dependent MSC senescence has been confirmed. MSCs ultimately cease growth when telomeres reach the length of 5.8-10.5 $\mathrm{kb}(51)$ and DNA damage occurs to the telomeric DNA (52). Telomerase prevents telomere erosion and induces telomere elongation by continuous restitution of the lost TTAGGG repeats at the chromosome termini (53), but the expression of telomerase in MSCs is undetectable throughout the entire cell lifespan $(54,55)$. The function of telomerase may relate to p53 (56) and TGF- $\beta 1$ (57), which are both significant repressors (58). Although telomere shortening is a hallmark of cell senescence, the length of telomeres varies from donor to donor, making telomere length an unreliable measure of MSC senescence. In addition, it can extent cell lifespan beyond the natural limit upon introduction of the telomerase catalytic subunit hTERT (59).

Epigenetic changes. Histone deacetylases (HDACs) are a class of enzymes that catalyze the removal of acetyl groups from the $\varepsilon$-amino group of lysine residues in the histone tail. HDACs can act as transcription regulators by sustaining the balance between the chromatin status of acetylated and deacetylated histones and regulate stem cell properties. Thus, the DNA status can be modulated as relaxed euchromatin forms, or condensed heterochromatin forms (60). MSCs undergo aging and spontaneous differentiation with the epigenetic dysregulation of histone $\mathrm{H} 3$ acetylation on $\mathrm{K} 9$ and $\mathrm{K} 14$, without affecting the methylation of their promoter sites (61). During the progression of MSC senescence, HDAC inhibitors were found to downregulate polycomb group genes (PcGs) 


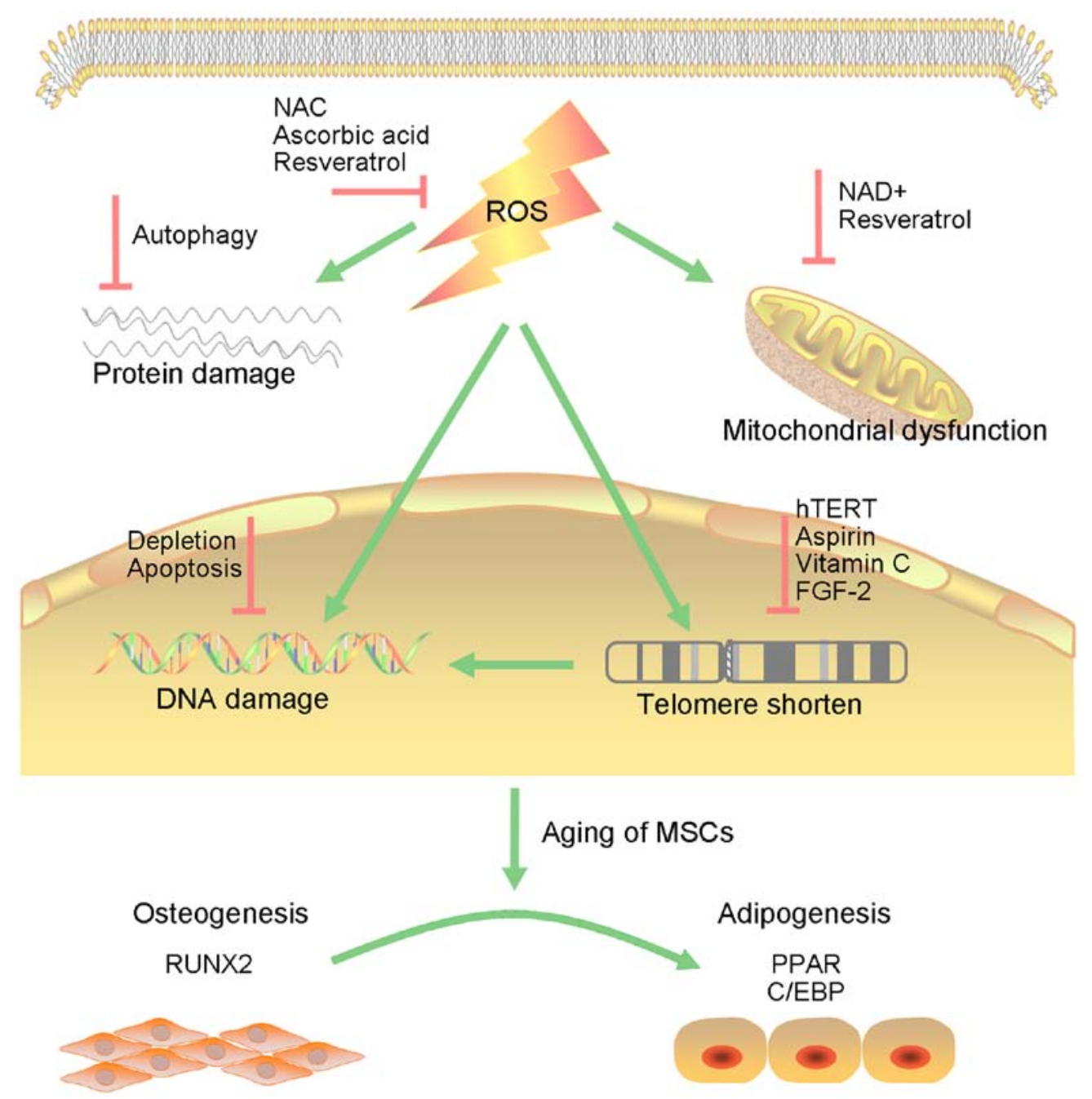

Figure 1. Common phenotypes in aging mesenchymal stem cells (MSCs) and the strategies by which to target and reverse these mechanisms. NAC, $N$-acetylL-cysteine; ROS, reactive oxygen species; PPAR, peroxisome proliferator-activated receptor; C/EBP, CCAAT/enhancer binding protein.

including BMI1, EZH2and SUZ12, and upregulate jumonji domain-containing 3 (JMJD3). The expression of EZH2 and SUZ12 was also regulated by the phosphorylation status of the retinoblastoma $(\mathrm{RB})$ protein following HDAC inhibitor treatment (62). HDAC inhibitors also activate a set of microRNAs (miRNAs) (let-7a1, let-7d, let-7f1, miR-23a, miR-26a and miR-30a) by altering the histone modification patterns within the vicinity of miRNA and RNA polymerase coding regions. Activated miRNAs strongly suppress the translation of high mobility group A2 (HMGA2), which in turn regulates cellular senescence genes, including $\mathrm{p} 16^{\mathrm{INK} 4 \mathrm{a}} / \mathrm{CDKN} 2 \mathrm{~A}$ (63). Sirtuin 1 (SIRT), an NADH-dependent protein deacetylase, plays a preventive role in many aging-associated disorders. It has been reported that SIRT1 expression is reduced in senescent MSCs, while its overexpression delays the onset of senescence and the loss of differentiation capacity (64).

The DNA methylation status of MSCs is proposed to be a better method for monitoring aging of MSCs. Gene expression can be regulated by DNA methylation through interference with transcription factors or methyl- $\mathrm{CpG}$ binding proteins, leading to the silencing of respective promoter regions (65). A correlation between histone acetylation and DNA methylation suggests that histone acetylation possibly determines DNA methylation (66). DNA methyltransferases (DNMTs) modulate the patterns of polycomb-mediated histone acetylation and methylation. Expression levels of DNMT1 and DNMT3B are significantly decreased during the replicative senescence of MSCs, leading to a decrease in the DNA methylation level, called hypomethylation, which is a distinct feature of senescent cells. In contrast, DNMT3a expression was found to be increased during replicative senescence, participating in the new methylation associated with senescence. DNMT inhibitors, such as 5-azacytidine, can upregulate cyclin-dependent kinase (CDK) inhibitors, p16 ${ }^{\mathrm{INK} 4 \mathrm{a}} / \mathrm{CDKN} 2 \mathrm{~A}, \mathrm{p} 21^{\mathrm{CIP} 1 / \mathrm{WAF} 1}$ and miRNAs targeting EZH1, and the induction of cellular senescence in MSCs (67).

Gene expression. The INK4a/ARF locus on chromosome 9p21 encodes two tumor-suppressor proteins, p16 ${ }^{\text {INK4a }} / C D K N 2 A$ and $\mathrm{p} 14 / \mathrm{p} 19^{\mathrm{ARF}}$, which are involved in growth arrest, cellular senescence and apoptosis in most mammalian tissues (68). In MSCs, p16 ${ }^{\mathrm{INK} 4 \mathrm{a}} / \mathrm{CDKN} 2 \mathrm{~A}-$ positive cells show growth retardation and increased activity of SA- $\beta$-gal. Furthermore, transfection of small interfering RNA (siRNA) targeting p16 ${ }^{\text {INK4a }} / C D K N 2 A$ in senescent MSCs results in the reduction of senescent cells and the ability of cells to maintain proliferation, suggesting 
that $\mathrm{p} 16^{\mathrm{INK} 4 \mathrm{a}} / \mathrm{CDKN} 2 \mathrm{~A}$ is an important regulator of MSC aging (69).

HMGA2 encodes a protein that belongs to the nonhistone chromosomal HMGA protein family. It consists of three DNA binding domains containing 8-9 amino acids (AA) and shows high affinity for short AT-rich sequence (70). As an architectural transcription factor, HMGA2 is involved in gene regulation. HMGA2 overexpression activates genes related to cell proliferation, such as cyclin A, cyclin F, cyclin E1 and CD25A (72). It also induces AKT phosphorylation and its downstream effectors in the mammalian target of rapamycin (mTOR)/p70S6K pathway, which is a serine/threonine protein kinase that regulates cell growth, cell proliferation and cell survival (71). The expression of HMGA2 decreases with age in MSCs, combined with the increased expression of p16 ${ }^{\mathrm{INK} 4 \mathrm{a}} / \mathrm{CDKN} 2 \mathrm{~A}, \mathrm{p} 19^{\mathrm{ARF}}, \mathrm{p} 21^{\mathrm{CIP} 1 / \mathrm{WAF} 1}$ and $\mathrm{p} 27^{\mathrm{KIP} 1}$ (73).

$\mathrm{RB}$ encodes the RB protein, which controls cell cycle progression from $\mathrm{G} 1$ into $\mathrm{S}$ phase by binding to $\mathrm{E} 2 \mathrm{~F}$ and inhibiting its activity (72). Recently, the mechanism of RB mediation was shown to play a positive role in regulating MSC properties by affecting DNA methylation via upregulation of DNA methyltransferase 1 (DNMT1) (73). In contrast, knockdown of the RB gene induces senescence (74). Thus, the RB gene is involved in the control of the MSC properties of aging and stemness (75).

The LMNA gene encodes nuclear lamina, which is composed of the A- and B-type lamins. Lamins A and C are members of the A-type lamins, which are derived from alternative splicing and are localized to the nuclear envelope and nucleoplasm. Lamins A and C can regulate important cellular events including DNA replication, cell division, transcriptional regulation and structural support (76). Usually, prelamin A, the precursor of lamin A, is catalyzed by ZMPSTE24/FACE-1, which is a membrane zinc metalloproteinase (77). Mutation of the LMNA gene produces progerin, which lacks the cleavage site for ZMPSTE24/FACE-1, resulting in the accumulation of farnesylated prelamin A. The accumulation of progerin and prelamin A leads to premature senescence characterized by nuclear blebbing, heterochromatin damage of HP1 and LAP2 and defects in DNA replication, transcription and repair (78). The introduction of progerin into MSCs interferes with cellular function by activating the major downstream effectors of the Notch signaling pathway and alters the differentiation potential (79). It has been reported that ZMPSTE24 expression is severely affected by replicative or HDAC inhibitor-mediated senescence in MSCs (80).

Immunological properties. Many cytokines and growth factors are secreted by MSCs and self-regulate their proliferation in culture, including interleukin-1 (IL-1), IL-3, IL-4, IL-6, IL-8, IL-17, epidermal growth factor (EGF), fibroblast growth factors-2 (FGF-2), FGF-4 and FGF-8, hepatocyte growth factor (HGF), insulin growth factor-1 (IGF-1), platelet-derived growth factor (PDGF), TGF- $\beta$ and vascular endothelial growth factor (VEGF) (81). These factors are called senescenceassociated secretory phenotype (SASP) factors (82), and some are crucial to MSC senescence as well. In addition, SASP mediated-inflammatory factors are also interconnected with inflammatory process. For example, in the presence of an inflammatory environment [high levels of tumor necrosis factor- $\alpha$ (TNF- $\alpha)$ and interferon- $\gamma$ (IFN- $\gamma)$ ], MSCs become activated through Toll-like receptor (TLR)4 (83) and adopt an immune-suppressive phenotype by secreting high levels of soluble factors, including IDO, PGE2, NO, TGF- $\beta$, HGF and hemoxygenase (HO) (84). Moreover, TNF- $\alpha$ and IFN- $\gamma$ activate CD106 expression in senescent MSCs. HO-1 degrades production of hemebiliverdin and carbon monoxide and exerts strong positive effects on osteogenesis while suppressing adipogenesis in MSCs (85). Thus, it plays a key factor as a stress-responsive, cytoprotective and immunoregulatory molecule.

The employment of exogenous TGF- $\beta$ expression was found to trigger premature cell senescence in MSCs confirming the role of TGF- $\beta$, with concomitant activation of $\mathrm{p} 16^{\mathrm{INK} 4 \mathrm{a}}$ and $\mathrm{p} 21^{\mathrm{CIP} 1}$ checkpoints (86). Analysis of the gene expression profile in senescent MSCs has shown that TGF- $\beta$ is increased in a dose-dependent manner (87). This effect coincided with upregulated SMAD3, a major signaling molecule for TGF- $\beta$. Inhibition of TGF- $\beta$ receptor signaling was found to promote the culture expansion of undifferentiatied MSCs (88).

\section{Strategies to prevent senescence}

For the use of MSCs in therapy, methods that allow the generation of large populations of MSCs without affecting their properties of differentiation or immunomodulation need to be established. The information described in this review may suggest a possible method to improve the therapeutic efficacy by regulating specific factors or the microenvironment associated with the MSC aging process. A number of senescent suppressors that function in the damage response or defense against oxidative stress are involved in modulation of the lifespan of human primary cells. Some of these suppressors, when overexpressed in human MSCs, induce substantial expansion of the proliferative potential and delay replicative senescence to an extent worthy of practical consideration.

The antioxidant $N$-acetyl-L-cysteine (NAC), a precursor of glutathione and a direct ROS scavenger, has been used as a therapeutic agent to ameliorate the damaging effects of ROS (89). Other antioxidants such as ascorbic acid and inhibitors of p38/MAPK or mTOR can markedly improve ROS-mediated injury in MSCs and lead to full recovery (90). NRF2 activation may also be an alternatively excellent strategy to extend the lifespan of BMSCs (91).

The introduction of hTERT into MSCs resulted in a substantial multiplication of their replicative lifespan accompanied by the preservation of a normal karyotype, elongation of telomeres and loss of the senescent phenotype without impact on differentiation ability $(92,93)$. Several small molecular compounds, such as aspirin and vitamin C, as well as FGF-2, have been developed to activate the endogenous telomerase of MSCs, achieving similar effects of improved proliferative and osteogenic potential in recent research (94). However, this is ill-advised for clinical applications given the small but possible risk of malignant transformation.

Genetic engineering of cells is one possible approach for preventing MSC aging (95). Knockdown of p16 $16^{\mathrm{INK} 4 \mathrm{a}}$ CDKN2A (96) or silencing of RB (75) in MSCs rescues the senescent phenotype and increases the proliferation rate and clonogenicity. However, silencing of these tumor-suppressorgenes disrupts differentiation potential and increases 
tumorigenesis risks. Knockdown or silencing of miR-195 significantly increases hTERT, phosphorylation of AKT and FOXO3 expression and induces telomere relengthening in senescent MSCs (97).

Selective growth factors have also been used to maintain the self-renewal and differentiation potential of MSCs. For instance, employing exogenous FGF-2, PDGF and EGF has been reported to increase proliferation ability and delay MSC senescence, without affecting osteogenesis and adipogenesis (98) for therapeutic use.

\section{Conclusion}

Recent studies have demonstrated promising results for the therapeutic utilization of MSCs in regenerative medicine. They show strong advantages and ability in cell therapy. However, the number of MSCs is usually $10^{8}-10^{10}$ for cell transplantation, which means a single cell in $10^{5}$ primary MSCs has to undergo 17 doublings and is evitable to consume its potential. The proliferative and functional activity of MSCs is destined to decline during the process of senescence, hindering the preparation of sufficient cells for clinical application. This calls for the investigation of the mechanisms of senescence and the development of efficient means to reverse it. This review provides a better understanding of the underlying mechanisms and significance of cellular senescence, facilitating ways to manipulate the replicative lifespan of MSCs. First, histological markers such as SA- $\beta$-gal and CFU-F, CD106 and STRO-1, as well as differentiation potential of osteogenesis and adipogenesis are used in detecting MSC aging and senescence. Second, the mechanisms of free radicals and oxidative stress on MSC senescence are illustrated. ROS and the consequent oxidative stress is at the base of aging. It leads to DNA damage, protein damage and mitochondrial dysfunction, which triggers the inner process of senescence, including changes in HDAC and DNA methyltransferase, imbalance of telomere and telomerase, genes and signaling pathways, as well as the secretory phenotype. Considering the above aspects, these strategies could be further investigated to prevent or reverse the MSC aging process.

\section{Acknowledgements}

This study was supported by the National Natural Scientific Foundations of China (no. 81200315).

\section{References}

1. Payushina O, Domaratskaya E and Starostin V: Mesenchymal stem cells: Sources, phenotype, and differentiation potential. Biol Bull 33: 2-18, 2006.

2. Musina RA, Bekchanova ES and Sukhikh GT: Comparison of mesenchymal stem cells obtained from different human tissues. Bull Exp Biol Med 139: 504-509, 2005.

3. Mareschi K, Ferrero I, Rustichelli D, Aschero S, Gammaitoni L, Aglietta M, Madon E and Fagioli F: Expansion of mesenchymal stem cells isolated from pediatric and adult donor bone marrow. J Cell Biochem 97: 744-754, 2006.

4. Zhang B, Liu R, Shi D, Liu X, Chen Y, Dou X, Zhu X, Lu C, Liang W, Liao L, et al: Mesenchymal stem cells induce mature dendritic cells into a novel Jagged-2-dependent regulatory dendritic cell population. Blood 113: 46-57, 2009.

5. Trivedi P and Hematti P: Derivation and immunological characterization of mesenchymal stromal cells from human embryonic stem cells. Exp Hematol 36: 350-359, 2008.
6. Anzalone R, Lo Iacono M, Corrao S, Magno F, Loria T, Cappello F, Zummo G, Farina F, La Rocca G: New emerging potentials for human Wharton's jelly mesenchymal stem cells: immunological features and hepatocyte-like differentiative capacity. Stem Cells Dev 19: 423-438, 2010.

7. Francese R and Fiorina P: Immunological and regenerative properties of cord blood stem cells. Clin Immunol 136: 309-322, 2010.

8. Patel DM, Shah J and Srivastava AS: Therapeutic potential of mesenchymal stem cells in regenerative medicine. Stem Cells Int 2013: 496218, 2013.

9. Rubin H: Promise and problems in relating cellular senescence in vitro to aging in vivo. Arch Gerontol Geriatr 34: 275-286, 2002.

10. Digirolamo CM, Stokes D, Colter D, Phinney DG, Class R and Prockop DJ: Propagation and senescence of human marrow stromal cells in culture: A simple colony-forming assay identifies samples with the greatest potential to propagate and differentiate. Br J Haematol 107: 275-281, 1999.

11. Campisi J and d'Adda di Fagagna F: Cellular senescence: When bad things happen to good cells. Nat Rev Mol Cell Biol 8: 729-740, 2007.

12. Stenderup K, Justesen J, Clausen C and Kassem M: Aging is associated with decreased maximal life span and accelerated senescence of bone marrow stromal cells. Bone 33: 919-926, 2003.

13. Zhou S, Greenberger JS, Epperly MW, Goff JP, Adler C, Leboff MS and Glowacki J: Age-related intrinsic changes in human bone-marrow-derived mesenchymal stem cells and their differentiation to osteoblasts. Aging Cell 7: 335-343, 2008.

14. Singh M and Piekorz RP: Senescence-associated lysosomal $\alpha$-L-fucosidase (SA- $\alpha$-Fuc): A sensitive and more robust biomarker for cellular senescence beyond SA- $\beta$-Gal. Cell Cycle 12: 1996, 2013.

15. Dominici M, Le Blanc K, Mueller I, Slaper-Cortenbach I, Marini F, Krause D, Deans R, Keating A, Prockop DJ and Horwitz E: Minimal criteria for defining multipotent mesenchymal stromal cells. The International Society for Cellular Therapy position statement. Cytotherapy 8: 315-317, 2006.

16. Yu Y, Park YS, Kim HS, Kim HY, Jin YM, Jung SC, Ryu KH and Jo I: Characterization of long-term in vitro culture-related alterations of human tonsil-derived mesenchymal stem cells: Role for $\mathrm{CCN} 1$ in replicative senescence-associated increase in osteogenic differentiation. J Anat 225: 510-518, 2014.

17. Simmons PJ and Torok-Storb B: Identification of stromal cell precursors in human bone marrow by a novel monoclonal antibody, STRO-1. Blood 78: 55-62, 1991.

18. Jung EM, Kwon O, Kwon KS, Cho YS, Rhee SK, Min JK and Oh DB: Evidences for correlation between the reduced VCAM-1 expression and hyaluronan synthesis during cellular senescence of human mesenchymal stem cells. Biochem Biophys Res Commun 404: 463-469, 2011.

19. Lv FJ, Tuan RS, Cheung KM and Leung VY: Concise review: The surface markers and identity of human mesenchymal stem cells. Stem Cells 32: 1408-1419, 2014.

20. Laschober GT, Brunauer R, Jamnig A, Fehrer C, Greiderer B and Lepperdinger G: Leptin receptor/CD295 is upregulated on primary human mesenchymal stem cells of advancing biological age and distinctly marks the subpopulation of dying cells. Exp Gerontol 44: 57-62, 2009.

21. Astudillo P, Ríos S, Pastenes L, Pino AM and Rodríguez JP: Increased adipogenesis of osteoporotic human-mesenchymal stem cells (MSCs) characterizes by impaired leptin action. J Cell Biochem 103: 1054-1065, 2008.

22. Atashi F, Modarressi A and Pepper MS: The role of reactive oxygen species in mesenchymal stem cell adipogenic and osteogenic differentiation: A review. Stem Cells Dev 24: 1150-1163, 2015.

23. Xu J, Qian J, Xie X, Lin L, Zou Y, Fu M, Huang Z, Zhang G, $\mathrm{Su} \mathrm{Y}$ and Ge J: High density lipoprotein protects mesenchymal stem cells from oxidative stress-induced apoptosis via activation of the PI3K/Akt pathway and suppression of reactive oxygen species. Int J Mol Sci 13: 17104-17120, 2012.

24. Jiang Y, Mishima H, Sakai S, Liu YK, Ohyabu Y and Uemura T: Gene expression analysis of major lineage-defining factors in human bone marrow cells: effect of aging, gender, and age-related disorders. J Orthop Res 26: 910-917, 2008.

25. Komori T: Signaling networks in RUNX2-dependent bone development. J Cell Biochem 112: 750-755, 2011.

26. Tu B,Peng ZX, Fan QM, Du L, Yan W and Tang TT: Osteosarcoma cells promote the production of pro-tumor cytokines in mesenchymal stem cells by inhibiting their osteogenic differentiation through the TGF- $\beta /$ Smad2/3 pathway. Exp Cell Res 320: 164-173, 2014. 
27. Xu C, Wang J, Zhu T, Shen Y, Tang X, Fang L and Xu Y: Cross-talking between PPAR and WNT signaling and its regulation in mesenchymal stem cell differentiation. Curr Stem Cell Res Ther 11: 247-254, 2016.

28. Isidori AM, Strollo F, Morè M, Caprio M, Aversa A, Moretti C, Frajese G, Riondino $\mathrm{G}$ and Fabbri A: Leptin and aging: Correlation with endocrine changes in male and female healthy adult populations of different body weights. J Clin Endocrinol Metab 85: 1954-1962, 2000.

29. Lee HM, Joo BS, Lee CH, Kim HY, Ock JH and Lee YS: Effect of glucagon-like peptide- 1 on the differentiation of adiposederived stem cells into osteoblasts and adipocytes. J Menopausal Med 21: 93-103, 2015.

30. Stringer B, Waddington R, Houghton A, Stone M, Russell G and Foster G: Serum from postmenopausal women directs differentiation of human clonal osteoprogenitor cells from an osteoblastic toward an adipocytic phenotype. Calcif Tissue Int 80: 233-243, 2007

31. Harman D. Aging: a theory based on free radical and radiation chemistry. J Gerontol 11: 298-300, 1956.

32. Poyton RO, Ball KA and Castello PR: Mitochondrial generation of free radicals and hypoxic signaling. Trends Endocrinol Metab 20: 332-340, 2009.

33. Balaban RS, Nemoto S and Finkel T: Mitochondria, oxidants, and aging. Cell 120: 483-495, 2005.

34. Bedard K and Krause K-H: The NOX family of ROS-generating NADPH oxidases: Physiology and pathophysiology. Physiol Rev 87: 245-313, 2007.

35. Reuter S, Gupta SC, Chaturvedi MM and Aggarwal BB Oxidative stress, inflammation, and cancer: How are they linked? Free Radic Biol Med 49: 1603-1616, 2010

36. Boutten A, Goven D, Boczkowski J and Bonay M: Oxidative stress targets in pulmonary emphysema: Focus on the Nrf2 pathway. Expert Opin Ther Targets 14: 329-346, 2010.

37. Jeong SG and Cho GW: Endogenous ROS levels are increased in replicative senescence in human bone marrow mesenchymal stromal cells. Biochem Biophys Res Commun 460: 971-976, 2015.

38. Benameur L, Charif N, Li Y, Stoltz JF and de Isla N: Toward an understanding of mechanism of aging-induced oxidative stress in human mesenchymal stem cells. Biomed Mater Eng 25 (Suppl 1) 41-46, 2015.

39. Stolzing A, Jones E, McGonagle D and Scutt A: Age-related changes in human bone marrow-derived mesenchymal stem cells: consequences for cell therapies. Mech Ageing Dev 129: $163-173,2008$

40. Tsai WB, Chung YM, Takahashi Y, Xu Z and Hu MC: Functional interaction between FOXO3a and ATM regulates DNA damage response. Nat Cell Biol 10: 460-467, 2008.

41. Milani P, Ambrosi G, Gammoh O, Blandini F and Cereda C: SOD1 and DJ-1 converge at Nrf2 pathway: a clue for antioxidant therapeutic potential in neurodegeneration. Oxid Med Cell Longev 2013: 836760, 2013.

42. Zhang F, Cui J, Liu X, Lv B, Liu X, Xie Z and Yu B: Roles of microRNA-34a targeting SIRT1 in mesenchymal stem cells. Stem Cell Res Ther 6: 195, 2015.

43. Bucciantini M, Giannoni E, Chiti F, Baroni F, Formigli L, Zurdo J, Taddei N, Ramponi G, Dobson CM and Stefani M Inherent toxicity of aggregates implies a common mechanism for protein misfolding diseases. Nature 416: 507-511, 2002.

44. Passos JF, Saretzki G, Ahmed S, Nelson G, Richter T, Peters H, Wappler I, Birket MJ, Harold G, Schaeuble K, et al: Mitochondrial dysfunction accounts for the stochastic heterogeneity in telomeredependent senescence. PLoS Biol 5: e110, 2007.

45. Hayflick L and Moorhead PS: The serial cultivation of human diploid cell strains. Exp Cell Res 25: 585-621, 1961.

46. Hwang ES: Senescence suppressors: Their practical importance in replicative lifespan extension in stem cells. Cell Mol Life Sci 71: 4207-4219, 2014.

47. Harley CB, Futcher AB, Greider CW. Telomeres shorten during ageing of human fibroblasts. Nature 345: 458-460, 1990.

48. Guillot PV, Gotherstrom C, Chan J, Kurata H and Fisk NM: Human first-trimester fetal MSC express pluripotency markers and grow faster and have longer telomeres than adult MSC. Stem Cells 25: 646-654, 2007.

49. Bonab MM, Alimoghaddam K, Talebian F, Ghaffari SH Ghavamzadeh A and Nikbin B: Aging of mesenchymal stem cell in vitro. BMC Cell Biol 7: 14, 2006.

50. Parsch D, Fellenberg J, Brümmendorf TH, Eschlbeck AM and Richter W: Telomere length and telomerase activity during expansion and differentiation of human mesenchymal stem cells and chondrocytes. J Mol Med (Berl) 82: 49-55, 2004.
51. Baxter MA, Wynn RF, Jowitt SN, Wraith JE, Fairbairn LJ and Bellantuono I: Study of telomere length reveals rapid aging of human marrow stromal cells following in vitro expansion. Stem Cells 22: 675-682, 2004.

52. Raz V, Vermolen BJ, Garini Y, Onderwater JJ, MommaasKienhuis MA, Koster AJ, Young IT, Tanke H and Dirks RW: The nuclear lamina promotes telomere aggregation and centromere peripheral localization during senescence of human mesenchymal stem cells. J Cell Sci 121: 4018-4028, 2008.

53. Masutomi K, Yu EY, Khurts S, Ben-Porath I, Currier JL, Metz GB, Brooks MW, Kaneko S, Murakami S, DeCaprio JA, et al: Telomerase maintains telomere structure in normal human cells. Cell 114: 241-253, 2003.

54. Graakjaer J, Christensen R, Kolvraa S and Serakinci N: Mesenchymal stem cells with high telomerase expression do not actively restore their chromosome arm specific telomere length pattern after exposure to ionizing radiation. BMC Mol Biol 8: 49, 2007.

55. Ryu E, Hong S,Kang J, Woo J,Park J,Lee J and Seo JS: Identification of senescence-associated genes in human bone marrow mesenchymal stem cells. Biochem Biophys Res Commun 371: 431-436, 2008.

56. Serakinci N, Christensen R, Graakjaer J, Cairney CJ, Keith WN, Alsner J, Saretzki G and Kolvraa S: Ectopically hTERT expressing adult human mesenchymal stem cells are less radiosensitive than their telomerase negative counterpart. Exp Cell Res 313: 1056-1067, 2007.

57. Sawada R, Ito $\mathrm{T}$ and Tsuchiya $\mathrm{T}$ : Changes in expression of genes related to cell proliferation in human mesenchymal stem cells during in vitro culture in comparison with cancer cells. J Artif Organs 9: 179-184, 2006.

58. Li H, Xu D, Li J, Berndt MC and Liu JP: Transforming growth factor $\beta$ suppresses human telomerase reverse transcriptase (hTERT) by Smad3 interactions with c-Myc and the hTERT gene. J Biol Chem 281: 25588-25600, 2006.

59. Bodnar AG, Ouellette M, Frolkis M, Holt SE, Chiu CP, Morin GB, Harley CB, Shay JW, Lichtsteiner S and Wright WE: Extension of life-span by introduction of telomerase into normal human cells. Science 279: 349-352, 1998.

60. Sengupta N and Seto E: Regulation of histone deacetylase activities. J Cell Biochem 93: 57-67, 2004.

61. Li Z, Liu C, Xie Z, Song P, Zhao RC, Guo L, Liu Z and Wu Y: Epigenetic dysregulation in mesenchymal stem cell aging and spontaneous differentiation. PLoS One 6: e20526, 2011.

62. Jung JW, Lee S, Seo MS, Park SB, Kurtz A, Kang SK and Kang KS: Histone deacetylase controls adult stem cell aging by balancing the expression of polycomb genes and jumonji domain containing 3. Cell Mol Life Sci 67: 1165-1176, 2010.

63. Lee S, Jung JW, Park SB, Roh K, Lee SY, Kim JH, Kang SK and Kang KS: Histone deacetylase regulates high mobility group A2-targeting microRNAs in human cord blood-derived multipotent stem cell aging. Cell Mol Life Sci 68: 325-336, 2011.

64. Yuan H-F, Zhai C, Yan X-L, Zhao DD, Wang JX, Zeng Q, Chen L, Nan X, He LJ, Li ST, et al: SIRT1 is required for long-term growth of human mesenchymal stem cells. J Mol Med (Berl) 90: 389-400, 2012

65. Jaenisch R and Bird A: Epigenetic regulation of gene expression: How the genome integrates intrinsic and environmental signals. Nat Genet 33 (Suppl): 245-254, 2003.

66. Cervoni N and Szyf M: Demethylase activity is directed by histone acetylation. J Biol Chem 276: 40778-40787, 2001.

67. So AY, Jung JW, Lee S, Kim HS and Kang KS: DNA methyltransferase controls stem cell aging by regulating BMI1 and EZH2 through microRNAs. PLoS One 6: e19503, 2011.

68. Rayess H, Wang MB and Srivatsan ES: Cellular senescence and tumor suppressor gene p16. Int J Cancer 130: 1715-1725, 2012.

69. Shibata KR, Aoyama T, Shima Y, Fukiage K, Otsuka S, Furu M, Kohno Y, Ito K, Fujibayashi S, Neo M, et al: Expression of the p16INK4A gene is associated closely with senescence of human mesenchymal stem cells and is potentially silenced by DNA methylation during in vitro expansion. Stem Cells 25: 2371-2382, 2007.

70. Grosschedl R, Giese K and Pagel J: HMG domain proteins: Architectural elements in the assembly of nucleoprotein structures. Trends Genet 10: 94-100, 1994.

71. Yu KR, Park SB, Jung JW, Seo MS, Hong IS, Kim HS, Seo Y, Kang TW, Lee JY, Kurtz A, et al: HMGA2 regulates the in vitro aging and proliferation of human umbilical cord blood-derived stromal cells through the mTOR/p70S6K signaling pathway. Stem Cell Res (Amst) 10: 156-165, 2013. 
72. Li Y, Nichols MA, Shay JW and Xiong Y: Transcriptional repression of the D-type cyclin-dependent kinase inhibitor p16 by the retinoblastoma susceptibility gene product $\mathrm{pRb}$. Cancer Res 54: 6078-6082, 1994

73. Lin SP, Chiu FY, Wang Y, Yen ML, Kao SY and Hung SC: $\mathrm{RB}$ maintains quiescence and prevents premature senescence through upregulation of DNMT1 in mesenchymal stromal cells. Stem Cell Rep 3: 975-986, 2014.

74. Alessio N, Bohn W, Rauchberger V, Rizzolio F, Cipollaro M, Rosemann M, Irmler M, Beckers J, Giordano A and Galderisi U: Silencing of RB1 but not of RB2/P130 induces cellular senescence and impairs the differentiation potential of human mesenchymal stem cells. Cell Mol Life Sci 70: 1637-1651, 2013.

75. Galderisi U, Cipollaro M and Giordano A: The retinoblastoma gene is involved in multiple aspects of stem cell biology. Oncogene 25: 5250-5256, 2006.

76. Hutchison CJ and Worman HJ: A-type lamins: Guardians of the soma? Nat Cell Biol 6: 1062-1067, 2004

77. Corrigan DP, Kuszczak D, Rusinol AE, Thewke DP, Hrycyna CA, Michaelis S and Sinensky MS: Prelamin A endoproteolytic processing in vitro by recombinant Zmpste24. Biochem J 387: $129-138,2005$.

78. Liu B, Wang J, Chan KM, Tjia WM, Deng W, Guan X, Huang JD, Li KM, Chau PY, Chen DJ, et al: Genomic instability in laminopathy-based premature aging. Nat Med 11: 780-785, 2005.

79. Scaffidi $\mathrm{P}$ and Misteli T: Lamin A-dependent misregulation of adult stem cells associated with accelerated ageing. Nat Cell Biol 10: 452-459, 2008

80. Yu KR and Kang KS: Aging-related genes in mesenchymal stem cells: A mini-review. Gerontology 59: 557-563, 2013.

81. Ksiazek K: A comprehensive review on mesenchymal stem cell growth and senescence. Rejuvenation Res 12: 105-116, 2009.

82. Rodier F and Campisi J: Four faces of cellular senescence. J Cell Biol 192: 547-556, 2011.

83. Liotta F, Angeli R, Cosmi L, Filì L, Manuelli C, Frosali F, Mazzinghi B, Maggi L, Pasini A, Lisi V, et al: Toll-like receptors 3 and 4 are expressed by human bone marrow-derived mesenchymal stem cells and can inhibit their T-cell modulatory activity by impairing Notch signaling. Stem Cells 26: 279-289, 2008.

84. Trento C and Dazzi F: Mesenchymal stem cells and innate tolerance: Biology and clinical applications. Swiss Med Wkly 140 w13121, 2010.

85. Barbagallo I, Vanella A, Peterson SJ, Kim DH, Tibullo D, Giallongo C, Vanella L, Parrinello N, Palumbo GA, Di Raimondo F, et al: Overexpression of heme oxygenase-1 increases human osteoblast stem cell differentiation. J Bone Miner Metab 28: 276-288, 2010.

86. Ito T, Sawada R, Fujiwara Y, Seyama Y and Tsuchiya T: FGF-2 suppresses cellular senescence of human mesenchymal stem cells by down-regulation of TGF-beta2. Biochem Biophys Res Commun 359: 108-114, 2007.
87. Wu J, Niu J, Li X, Wang X, Guo Z and Zhang F: TGF- $\beta 1$ induces senescence of bone marrow mesenchymal stem cells via increase of mitochondrial ROS production. BMC Dev Biol 14: 21, 2014.

88. Gurung S, Werkmeister JA and Gargett CE: Inhibition of transforming growth factor- $\beta$ receptor signaling promotes culture expansion of undifferentiated human endometrial mesenchymal stem/stromal cells. Sci Rep 5: 15042, 2015.

89. Lin TM, Tsai JL, Lin SD, Lai CS and Chang CC: Accelerated growth and prolonged lifespan of adipose tissue-derived human mesenchymal stem cells in a medium using reduced calcium and antioxidants. Stem Cells Dev 14: 92-102, 2005.

90. Choi KM, Seo YK, Yoon HH, Song KY, Kwon SY, Lee HS and Park JK: Effect of ascorbic acid on bone marrow-derived mesenchymal stem cell proliferation and differentiation. J Biosci Bioeng 105: 586-594, 2008.

91. Su ZY, Shu L, Khor TO, Lee JH, Fuentes F and Kong AN: A perspective on dietary phytochemicals and cancer chemoprevention: oxidative stress, nrf2, and epigenomics. Top Curr Chem 329: 133-162, 2013

92. Takeuchi M, Takeuchi K, Kohara A, Satoh M, Shioda S, Ozawa Y, Ohtani A, Morita K, Hirano T, Terai M, et al: Chromosomal instability in human mesenchymal stem cells immortalized with human papilloma virus E6, E7, and hTERT genes. In Vitro Cell Dev Biol Anim 43: 129-138, 2007.

93. Simonsen JL, Rosada C, Serakinci N, Justesen J, Stenderup K, Rattan SI, Jensen TG and Kassem M: Telomerase expression extends the proliferative life-span and maintains the osteogenic potential of human bone marrow stromal cells. Nat Biotechnol 20: 592-596, 2002

94. Wei F, Qu C, Song T, Ding G, Fan Z, Liu D, Liu Y, Zhang C, Shi $S$ and Wang S: Vitamin C treatment promotes mesenchymal stem cell sheet formation and tissue regeneration by elevating telomerase activity. J Cell Physiol 227: 3216-3224, 2012.

95. Gharibi B, Farzadi S, Ghuman M and Hughes FJ: Inhibition of $\mathrm{Akt} / \mathrm{mTOR}$ attenuates age-related changes in mesenchymal stem cells. Stem Cells 32: 2256-2266, 2014

96. Gu Z, Cao X, Jiang J, Li L, Da Z, Liu H and Cheng C: Upregulation of p16INK4A promotes cellular senescence of bone marrow-derived mesenchymal stem cells from systemic lupus erythematosus patients. Cell Signal 24: 2307-2314, 2012.

97. Okada M, Kim HW, Matsu-Ura K, Wang YG, Xu M and Ashraf M: Abrogation of age-induced MicroRNA-195 rejuvenates the senescent mesenchymal stem cells by reactivating telomerase. Stem Cells 34: 148-159, 2016.

98. Gharibi B and Hughes FJ: Effects of medium supplements on proliferation, differentiation potential, and in vitro expansion of mesenchymal stem cells. Stem Cells Transl Med 1: 771-782, 2012. 